\title{
Using WReSTT Cyberlearning Environment in the Classroom
}

\section{Dr. Peter J Clarke, Florida International University}

Peter J. Clarke received his B.Sc. degree in Computer Science and Mathematics from the University of the West Indies (Cave Hill) in 1987, M.S. degree from SUNY Binghamton University in 1996 and Ph.D. in Computer Science from Clemson University in 2003. His research interests are in the areas of software testing, software metrics, model-driven software development, domain-specific modeling languages, and computer science education. He is currently an associate professor in the School of Computing and Information Sciences at Florida International University. He is a member of the ACM (SIGSOFT, SIGCSE, and SIGAPP); IEEE Computer Society; and a member of the Association for Software Testing (AST).

\section{Dr. Debra Lee Davis, Florida International University}

Dr. Debra Davis is an Instructor in the School of Computing and Information Sciences at Florida International University. Her research interests emphasize interdisciplinary topics including understanding and improving: (1) Computer Science education, including increasing participation of women; (2) educational applications and techniques for online STEM learning; and (3) complex human-machine interactions. She has a Ph.D. and M.A., in Cognitive Developmental Psychology from the University of Texas at Austin, and an M.S. in Computer Science from FIU.

\section{Mr. Raymond Chang Lau, Florida International University}

I am a software engineer with over 3 years experience in the development of web/mobile applications and services. Graduated Florida International University with a B.S. and M.S. in computer science.

\section{Dr. Yujian Fu P.E., Alabama A\&M University}

Dr. Yujian Fu is an associate professor of computer science department at Alabama A\&M University. Her research interests fall in formal verification of cyber physical systems, behavioral analysis of mobile security, software architecture and design analysis of safety-critical and mission-critical systems. Her projects are supported by NSF, Air Force and DoD. She have several publications regarding to the research and educational projects.

\section{Dr. James D Kiper, Miami University}

James Kiper is Chair and Professor of the Department of Computer Science and Software Engineering at Miami University. He teaches a variety of courses across the CS and SE curricula. His research is in the areas of software testing, software risk assessment, design rationale, and computer science and software engineering teaching and learning.

\section{Dr. Gursimran Singh Walia, North Dakota State University}

Gursimran S. Walia is an associate professor of Computer Science at North Dakota State University. His main research interests include empirical software engineering, software engineering education, human factors in software engineering, and software quality. He is a member of the IEEE Computer Society. Contact him at gursimran.walia@ndsu.edu 


\title{
Using WReSTT Cyberlearning Environment in the Classroom
}

\begin{abstract}
The impact of developing high quality software in todays society cannot be under estimated. Testing continues to be the main approach to ensure the quality of software during development. However, many academic institutions that teach computer science do not teach any software testing courses in their undergraduate or graduate curricula. In this paper we describe a cyberlearning tool (WReSTT-CyLE Web-Based Repository of Software Testing Tutorials: a Cyberlearning Environment) that helps students and instructors to learn various software testing techniques and testing tools. We also report on the results of a four year project that aimed to (1) train instructors in the use of various software testing techniques and tools, and (2) support pedagogy by using WReSTT-CyLE to expose students to several software testing techniques and testing tool tutorials. WResTT-CyLE contains learning material on software testing, and uses a combination of learning and engagement strategies (LESs) to get students more involved in the learning process.
\end{abstract}

\section{Introduction}

Most systems used in everyday life contain software; these systems include mobile phones, automobiles, and medical devices, among others. It is becoming more important to ensure that the software in these systems is properly developed before the system is released. Computer science and information technology (CS/IT) academics and practitioners agree that a comprehensive strategy to improve the number and quality of the 21 st century software development workforce is needed. One of the skills this workforce needs is the ability to develop high quality software. The main approach used to ensuring the quality of software during development is continuous testing of the software using the appropriate testing techniques and testing tools.

In this paper we describe a cyberlearning tool, WReSTT-CyLE Web-Based Repository of Software Testing Tutorials: a Cyberlearning Environment (http://wrestt.cis.fiu.edu/), that helps students and instructors to learn various software testing techniques and testing tools. We report the results of a four year multi-institution project that aimed to (1) train instructors in the use of various software testing techniques and tools, and (2) support pedagogy by using the cyberlearning tool to expose students to several software testing techniques and testing tools. Unlike other learning management systems, WReSTT-CyLE uses a combination of learning and engagement strategies (LESs) to get students more involved in the learning process. The learning content in WResTT-CyLE during the project focused on software testing concepts and testing tools. The institutions involved in the project are Florida International University (FIU), Alabama A\&M University (AAMU), Miami University (MU) and North Dakota State University (NDSU).

The LESs used in WReSTT-CyLE include collaborative learning, gamification and social interaction. The contribution of this work is contained in the presentation on the following items: (1) briefly introduce WReSTT-CyLE including how the learning materials are structured, and the implementation of the LESs; (2) report on the instructor workshops that were held during the project; (3) report on the results of instructor surveys conducted after the workshops; and (3) report on 
the results of a multi-institution student survey. In this paper we focus more on a description of the LESs used in WReSTT-CyLE, the representation of the learning materials (Digital Learning Objects) the results of the instructor workshops, and the results of the surveys conducted during the course of the project. Two survey questionnaires were administered during the studies where we asked instructors and students about self-reported experience of the workshops and WReSTTCyLE, respectively.

\section{Background}

In this section we briefly introduce digital learning objects (DLOs), learning and engagement strategies (LESs) and testing concepts and tools as they relate to WReSTT-CyLE.

\subsection{Digital Learning Objects}

A learning object is any entity, digital or non-digital, that may be used for learning, education or training. ${ }^{11}$ In our project we restrict learning objects to the digital form (Digital Learning Object - DLO). DLOs are very attractive to the project because they are much smaller chunks of learning content than courses, modules or units; they usually require between 2 to 15 minutes for completion; and they are self-contained interactive, reusable and can be aggregated. ${ }^{19}$ The IEEE standards for LOs identify four components for each LO: (1) Objective - should address only one learning objective; (2) Content - learning material focuses on that objective; (3) Practice - opportunity for learners to review facts and key concepts using: exercises and/or quiz-type self-tests; and (4) Assessment - the LO should assess whether the learner has achieved the learning objective.

\subsection{Learning and Engagement Strategies}

The learning and engagement strategies used in the WReSTT-CyLE project include collaborative learning, gamification and social interaction. Collaborative learning is where two or more people work in groups mutually searching for understanding, solutions, or meanings, or creating a product. ${ }^{18}$ Collaborative learning promotes several education goals, including: involvement, cooperation and teamwork and civic responsibility. ${ }^{18}$ Gamification uses game design elements and game mechanics to improve user experience and engagement with a system, ${ }^{14}$ which can be applied to an educational context. Importantly, gamification is not about adding "games" to a system. It is about taking elements commonly implemented in games that humans find fun and enjoyable, and applying those to other domains resulting in increased engagement. Social Interaction is an approach that enhances knowledge acquisition using various social activities. There is evidence in the literature that suggest social interaction can improve students' educational experiences. ${ }^{13}$

\subsection{Testing Concepts}

Testing continues to be one of the most used approaches to verify software. Software testing is defined as the dynamic verification of the behavior of a program on a finite set of test cases, suitably selected from the usually infinite executions domain, against the expected behavior. ${ }^{1}$ The most common testing approaches are black-box and white-box testing. Black-box testing techniques generate test cases based on the specification of the component, while white-box testing techniques guide the generation of test cases based on properties of the implementation. ${ }^{15}$ Based on the various approaches used to test software, testing tools have been developed to help automate the testing 
process. Examples of these tools are: IBM Rational Functional Tester ${ }^{10}$ - system testing (blackbox testing); JUnit ${ }^{8}$ - unit and subsystem testing (black-box testing); and EclEmma ${ }^{9}$ - unit and subsystem testing (white-box testing).

\section{WReSTT-CyLE}

In this section we describe the high-level architecture of WReSTT-CyLE focusing on how the LESs are integrated into WReSTT-CyLE. We also show a screen shot of an interface used by students to illustrate the usability of the system. It should be noted that WReSTT-CyLE does not replace existing learning management systems such as Moodle. ${ }^{16}$ Unlike Moodle, WReSTT-CyLE does not provide the facilities to maintain a gradebook, or post weekly assignments. We foresee students accessing WReSTT-CyLE by embedding a link in the course pages in Moodle.

\subsection{WReSTT-CyLE Architecture}

WReSTT-CyLE is an ongoing project for the past eight years and during that time there has been three versions of the system. Clarke et al. ${ }^{4}$ describes the first two versions of WReSTT. Version 3 of the system integrate the LESs into the various features of the system. WReSTT-CyLE continues to use the four-tier architecture, as in previous versions, and is implemented using Drupal 8. ${ }^{5}$ Figure 1 shows a block diagram of the main features in WReSTT-CyLE. These features include:

- Authentication - provides various users with different levels of access. These users include students, instructors and administrators.

- LESs - the learning and engagement strategies supported in WReSTT-CyLE including collaborative learning, gamification and social interaction. Additional details on the integration of the LESs are described later in the text.

- Learning Content - contains the digital learning objects (DLOs) on various testing topics and tutorials for several testing tools.

- Administration - provides the administrator with the ability to generate various reports (number of users, types of users, etc.), configure the system, and coordinate the course management activities.

- Course Management - generate student reports for instructors and individual students, assignment of student and instructor roles.

Figure 2 shows the student's landing screen for the a course (Mock Course). In the upper left of the screen there are two buttons that allow students to browse DLOs and tutorials in the WReSTTCyLE repository. The middle of the page shows the DLOs the student have been assigned and the progress made by both the student and his/her team. The bottom left of the screen shows the other members of the team, and to the right the student's activity stream. The student can also view his/her virtual points either using a graphical view or a tabular view.

\subsection{Integrating LESs into WReSTT-CyLE}

Currently there are three LESs integrated into the various features of WReSTT-CyLE, collaborative learning, gamification and social interaction. Collaborative learning is centered around virtual teams and/or the entire class participating in various WReSTT-CyLE activities. Collaborative learning occurs as follows: (1) all members in a virtual team are required to complete a DLO 


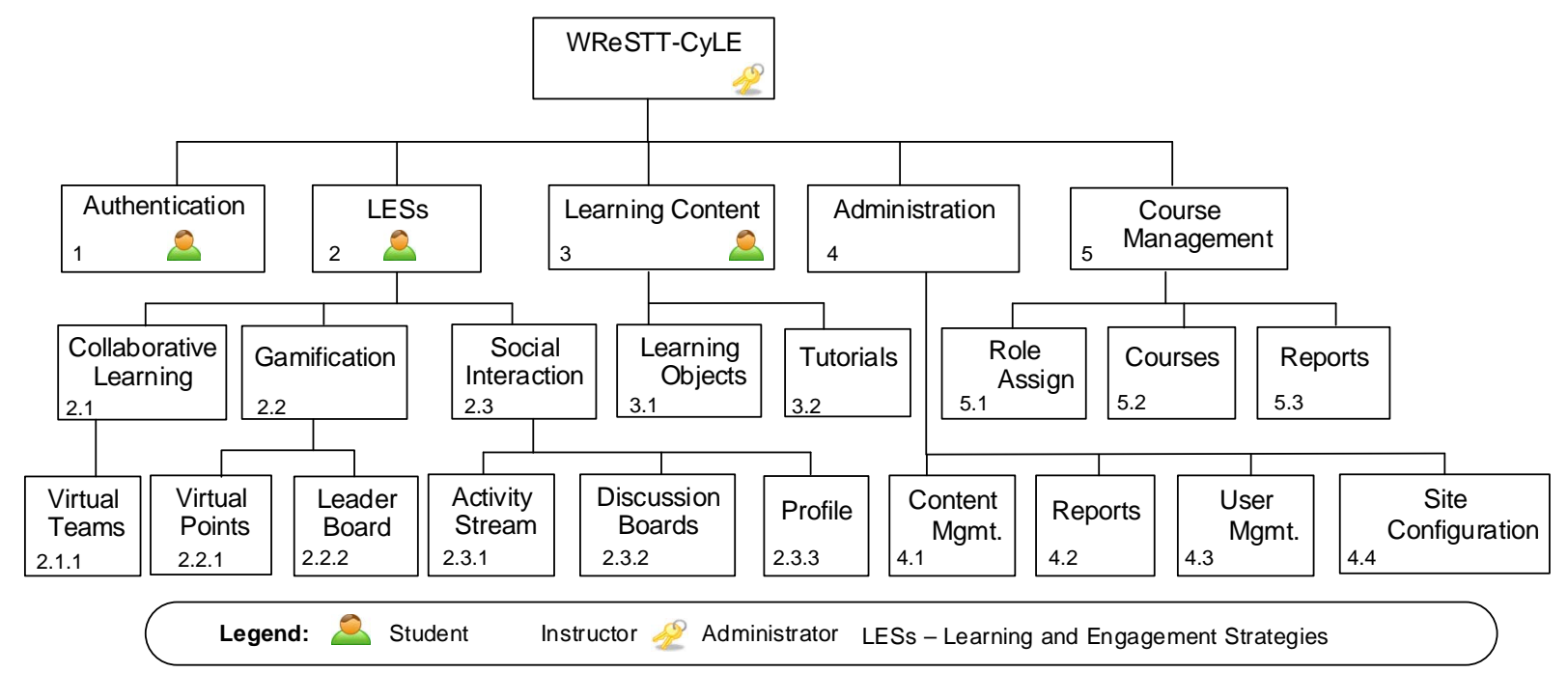

Figure 1: Block diagram of WReSTT version 3.

before each student's profile is updated to indicate the DLO has been completed; and (2) team member post to the class forum in WReSTT-CyLE benefiting other students in the class. Figure 2 shows how the progress of the assigned DLOs are displayed (both individual and team).

Gamification in WReSTT-CyLE is based on a virtual points system. These virtual points are awarded to each student upon successful completion of assigned individual and team activities, or other activities designed to motivate students to interact with WReSTT-CyLE. The virtual points are displayed in a leader board for the top five students in the class. Students may be awarded virtual points based on the following activities: individual - completing DLOs, viewing tutorials, posting to the class forum, updating their user profile; and collaborative - completing DLOs and posting helpful learning content that benefits the class. Although not shown in the student's screen in Figure 2, the actual screen does contain the class (course) leader board and the class wide (course) activity stream.

Social interaction is based on the use of various social features during the learning process, either individually or as part of a learning community. These features include: student profiles, message forums, rating of DLOs, rating of tutorials, rating of forum posts, activity streams (individual and class wide). Figure 2 shows the student's course activity stream and the pictures of the other team members, taken from the team members' profile. We recommend that instructors use virtual points as a very small percentage of the overall course grade or not at all.

\section{Using WReSTT-CyLE in the Classroom}

The development of WReSTT-CyLE was part of a larger project to integrate testing into advanced CS/IT courses. Two of the key objectives of the project were: (1) to develop faculty expertise in software testing and in using the new features in WReSTT-CyLE to support pedagogy through conduct bi-annual workshops, and (2) foster students' acquisition of software testing concepts and skills in upper-level undergraduate courses with a programming component. In this section we describe the training instructors received during the project and present a summary of the metrics for the workshops conducted. 


\section{Course: Mock Course}

\section{Learning Materials \\ Browse Tool Tutorials}

Browse Learning Objects

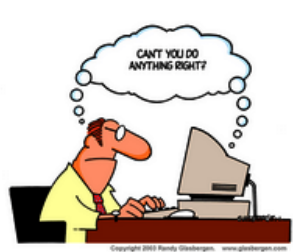

Assigned Learning Objects

\section{Introduction to Software Testing}

Subject: General Software Testing

My Progress

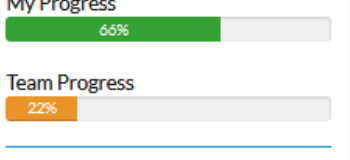

Open

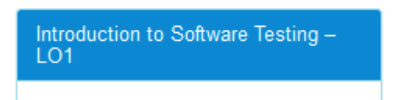

Subject: Software Testing

My Progress

My Progress
6696

Team Progress

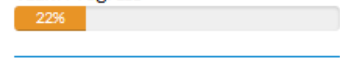

Open

\section{Introduction to Software Testing -
LO2}

Subject: Software Testing

My Progress

$66 \%$

Team Progress

Team Progress
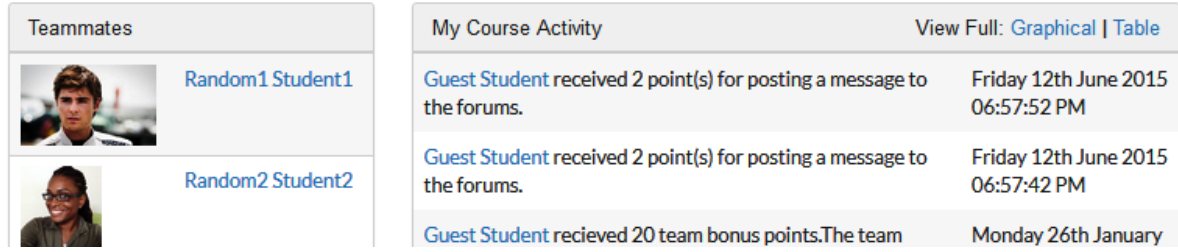

Figure 2: Student course access page in WReSTT-CyLE.

\subsection{Professional Development Supported by WReSTT-CyLE}

During the project five (5) workshops were conducted, four were conducted at Florida International University (FIU) and one was conducted at the Special Interest Group on Computer Science Education (SIGCSE) annual conference. Four of the workshops were titled Workshop on Integrating Software Testing into Programming Courses (WISTPC) these workshops occurred in 2013 (WISTPC-13), 2104 (WISTPC-14:1 at SIGCSE and WISTPC-14:2) and 2015 (WISTPC-15). The final workshop was Workshop on Using Learning and Engagement Strategies in Software Engineering and Programming Courses (LESSEP) 2016 (LESSEP-16). The objectives of the WISTPC workshops were:

- Provide a forum where CS/IT instructors can improve their knowledge of software testing and software testing tools to support pedagogy.

- Learn how to use WReSTT-CyLE in the classroom, including the collaborative learning, social networking and gamification features.

- Explore pedagogical techniques that can be used to integrate software testing into existing 
courses using the features and learning materials provided by WReSTT-CyLE.

The objectives of the LESSEP workshop was similar to the WISTPC workshops, except that the focus was on the LESs and the development of learning objects for software engineering and programming courses. In this paper we focus on the WISTPC workshops at FIU. We have recently started the LESSEP workshops and are still collecting data.

The WISTPC workshops were held over one and a half days consisting of 11 working sessions. These sessions included: two (2) sessions on software testing, two (2) sessions on the introduction to the students' view of WReSTT-CyLE, two (2) sessions on the introduction to the instructors' view of WReSTT-CyLE, one (1) session on using DLOs in WReSTT-CyLE, two (2) sessions on designing and conducting a research study, and two (2) sessions on using software testing tools in the classroom. Additional details of the workshops can be found at http://wrestt.cis.fiu.edu/events.

On average for each WISTPC workshops held at FIU, there were 19 applications for the workshops, 14 applicants attended the workshops, 27 total attendees (including PIs and students) attended the workshops, and 18 institutions were represented at the workshops. Based on the surveys conducted at the workshops the attendees found the workshops to be very helpful, relevant to their courses and interesting. This was reflected in attendees ratings and feedback. More specifically, the attendees indicated that they found the content and design of the workshops to be (on a 5 point scale): objectives were communicated appropriately (4.5), lived up to their expectations (4.5), relevant to their programming courses (4.6). will help improve their courses (4.4), showed how testing can be integrated into their existing courses (4.3), and stimulated learning (4.7).

The average of the survey results conducted at the the WISTPC workshops related to WReSTTCyLE are as follows: the likelihood the attendees would encourage students to use the resources in WReSTT-CyLE (3.3), the frequency they would use the resources in WReSTT-CyLE (3.1); and the likelihood they would encourage colleagues to attend the workshop (3.7). These low ratings may have resulted from the number of learning objects (DLOs) currently in WReSTTCyLE, and more specifically, the DLOs are not targeted to the CS1/2 but more focused to the software engineering and software testing courses. Many of the participants at the workshops taught CS1/2 courses.

\subsection{How WReSTT-CyLE may be Used in the Classroom}

Several of the authors of this paper have used WReSTT-CyLE in the classroom for classes involving programming or software testing. These classes include undergraduate and graduate software engineering and software testing, and CS1/2. There are currently several papers in the literature that report on how WReSTT-CyLE has been used in the classroom for each of the courses listed above, as well as the results obtained from the respective studies. ${ }^{3,4,7}$ Clarke at al. ${ }^{4}$ describe the educational impact using WReSTT in two editions of Software Engineering courses at FIU. This impact included knowledge gains in both the use of software testing techniques and testing tools. Based on the statistical analysis the treatment groups (students using WReSTT) showed significant gains over the control groups (student not using WReSTT) in both the pre/posttest administered and the use of tools in the class project.

In this paper we present a generic approach that seems to work best based on the feedback provided at the workshops described in Section 4.1 and the published papers. We advocate using a minimally 
disruptive approach when introducing WReSTT-CyLE in the classroom. That is, we do not expect WReSTT-CyLE to replace existing learning management systems such as Moodle or Blackboard already in use, or significantly change the content of the syllabus or the instructor's teaching style. We recommend the following steps instructors may follow when using WReSTT-CyLE in the classroom. We assume that the instructor is familiar with the learning content - DLOs and tutorials available in WReSTT-CyLE.

1. Prior to the beginning of the semester request a course instance from the WReSTT-CyLE system administrator.

2. During the course setup perform the following:

(a) Decide on the combination of LESs to use in the class (see Section 3.2)

(b) Upload the class roll

(c) Assign the virtual teams, assuming collaborative learning is being used.

(d) Assign virtual points to each tasks, assuming gamification is being used

3. Encourage students to review the help videos after logging into the system.

4. Provide students with guidance on how points will be awarded, assuming gamification will be used.

5. During the semester assign DLOs and tutorials to be reviewed by the students.

6. Monitor how students are interacting with the system and their performance on the DLOs.

7. At the end of the semester (or when needed) download the virtual points and use them as appropriate. We recommend that the virtual points be used as a very small percentage of the course grade if used at all.

We expect that instructors and students will benefit from using WReSTT-CyLE in the following ways. Instructors will have access to learning content that has been vetted and can be easily accessed. We do not recommend that the content in WReSTT-CyLE replace the content currently used by instructor's in their classes but supplement the existing content. Students can benefit from using WReSTT-CyLE by easily accessing testing tool tutorials located in one place thereby reducing the time to find such resources.

\section{Case Study}

In this section we report the results of surveys instruments completed by instructors and students who have used WReSTT-CyLE in the classroom. The instructor survey results are the first postworkshop results presented to the community and the student results represent the cumulative results across the four institutions participating in the project. The initial project ourcomes required that (1) we conduct post-workshop surveys to determine the usefulness and impact WReSTT-CyLE has on classes taught by the instructors and the ease of integrating testing into the upper-level courses, and (2) increase students' exposure to the software testing learning content and tutorials contained in WReSTT-CyLE. The specific objectives of the study are to determine if:

1. Instructor: Attending the WISTPC workshop had any impact on the classes taught, specifically integrating testing into my classes.

2. Instructor: Attending the WISTPC workshop encouraged the use of WReSTT-CyLE in my class.

3. Student: WReSTT-CyLE provided a positive user experience.

4. Student: The software testing learning content (DLOs and tool tutorials) is adequate. 


\subsection{Methods}

Sample: The sample consisted of both instructors and students for the years 2013 to 2016. The instructors were selected from attendees at the WISTPC-13, WISTPC-14:2 and WISTPC-15 workshops at FIU. The total number of instructors attending the workshops were 46 from 33 different academic intuitions. Based on the data collected from WReSTT-CyLE there were 1245 students registered in WReSTT-CyLE during the three year period.

Data Collection: Data for the study was collected from instructor and student survey questionnaire instruments, as well as data collected in WReSTT-CyLE for the years 2012 to 2015. These instruments were administered using Qualtrics ${ }^{17}$ with a cover page providing the participants with the ability to opt out of the survey. To date the student survey has been administered twice, Fall 2015 and Spring 2016, and the instructor survey once in Fall 2015.

Design: The instruments used in this study were survey questionnaires administered via the internet using Qualtrics. Content and face validity of both instruments was validated by a group of internal and external evaluators who have expertise in survey development, STEM education initiatives and user experience research. This involved a series of iterative evaluations done by each expert prior to Fall 2015. Although we conducted other studies using various designs, ${ }^{3,4}$ the design for this study was based mainly on using survey questionnaires to capture the data. The survey questions were based around the objectives of the study presented in the introduction to this section.

In order to administer the instructor surveys, email addresses were aggregated from the WISTPC13, WISTPC-14:2 and WISTPC-15 workshops attendance records. These email addresses were then used to send out the survey instruments. The email addresses for the student surveys were obtained from WReSTT-CyLE for the three years being considered. The survey participants were given two weeks to respond to the request to complete the survey. To obtain the data from WReSTT-CyLE the system administrator queried the various databases contained in WReSTTCyLE. A total of 46 instructor survey requests were sent out targeting the instructors that attended the WISTPC-13, WISTPC-14:2 and WISTPC-15 workshops. Of the 46 requests sent out 20 surveys were completed (43.5\%). In Fall 2015 and Spring 2016 a total of 1055 student survey requests were sent out and 58 surveys were completed $(5.5 \%)$.

\subsection{Results and Analysis}

Based on the survey responses $56 \%$ of the instructors who had attended the workshops indicated that they had subsequently used WReSTT-CyLE in their courses. Overall, instructors rated the workshop as useful and relevant for their courses. Ratings for questions regarding the workshops impact ranged from 3.47 to 4.21 (out of 5), with all but one question receiving an average rating of at least 4.0. Instructors generally viewed the workshops as helping them acquire new knowledge relevant to their course work, with ratings on knowledge acquisition ranging from 3.89 to 4.21 (out of 5), with all but one question receiving and average rating of at least 4.05. These results are illustrated in Figure 3.

A majority of instructors who completed the survey indicated that they at least sometimes used WReSTT-CyLE in their courses $(80 \%)$ and that they found the use of WReSTT-CyLE helpful (60\%). While WReSTT-CyLE was not often used in the instructors' research, the majority of 


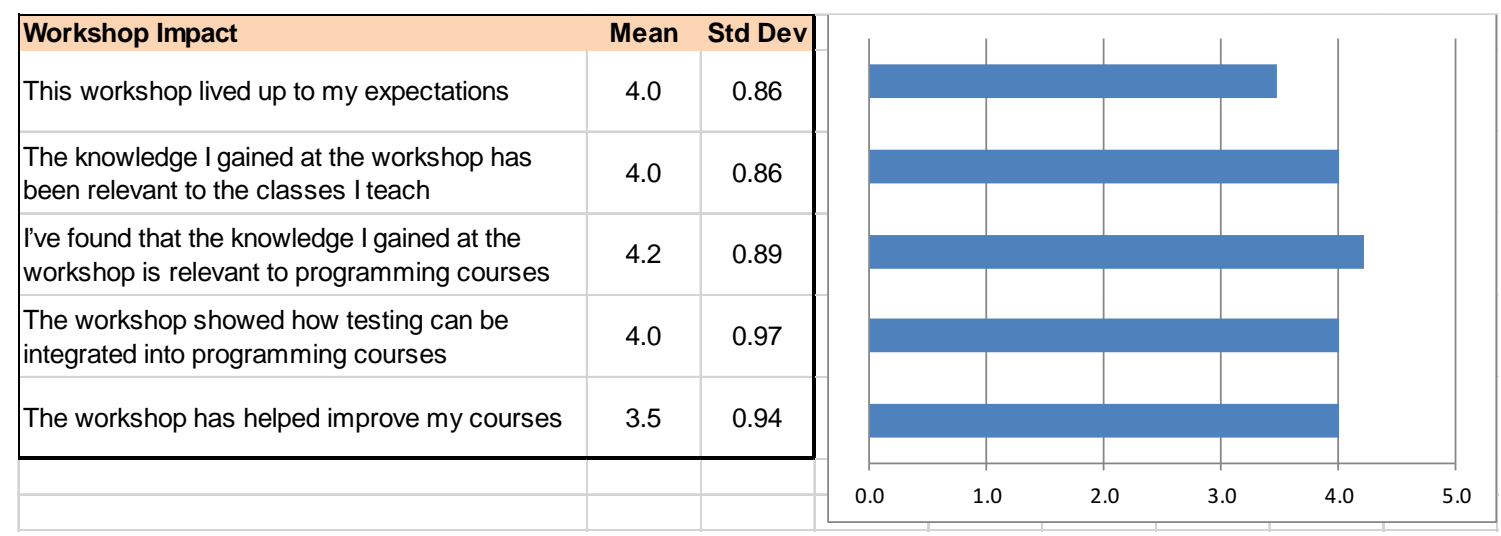

Figure 3: Table showing partial results of the instructors' survey.

instructors indicated that they used WReSTT-CyLE in their course preparation $(87.5 \%)$ and found it to be helpful (75\%). Finally, 75.7\% of instructors indicated that WReSTT-CyLE might be useful for their colleagues as a teaching resource, and, as a result of their exposure to WReSTT, 68.4\% indicated that they would encourage your colleagues to integrate software testing in their relevant courses.

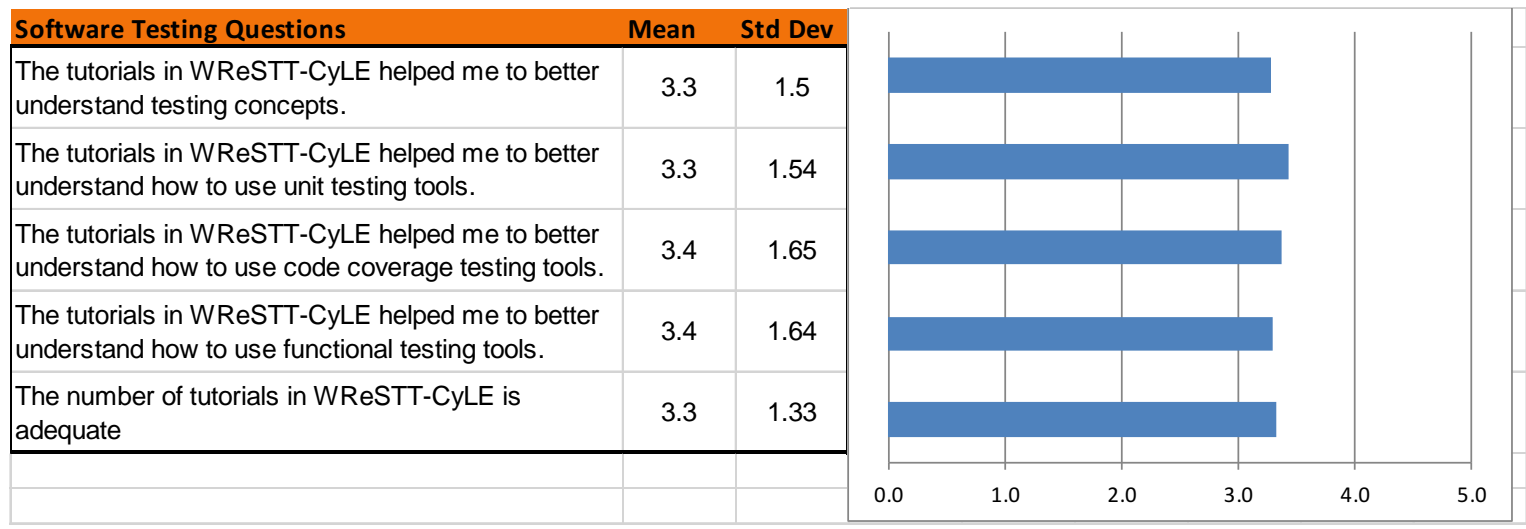

Figure 4: Table showing partial results of the students' survey.

Based on the responses of the student surveys $67 \%$ of students had not previously used a learning resource other than WReSTT-CyLE to learn about testing concepts or testing tools. Overall, students rated the new site as user friendly and with good functionality. Ratings for questions regarding user experience and usability ranged from 3.10 to 3.94 (out of 5). Feedback also included numerous comments on how to improve the site. The primary requested improvements revolved around improved navigation and the inclusion of an online IDE.

Students generally viewed the software testing tutorials available in WReSTT-CyLE as good, with ratings for questions regarding the tutorials ranging from 3.28 to 3.44. Students indicated that greater improvements in this area would be realized if a greater number of tutorials were included in the site, along with more interactive activities as part of the learning process. These results are illustrated in Figure 4. It should be noted that in the survey we referred to both the DLOs and tool tutorials as tutorials.

Students generally viewed the collaborative learning approaches used in WReSTT positively, it encouraged greater engagement in the learning process. Student ratings of the collaborative learning 
approaches used in WReSTT-CyLE ranged from 3.36 to 3.59 (out of 5). The highest score (3.59) in this section of the survey was in response to the statement: The event stream showing the activities of the other members in the class encouraged my team to complete my tasks in WReSTT. This statement incorporates collaborative learning and social interaction. The following statement had one of the highest scores (3.48): The use of virtual points in WReSTT encouraged me to visit the website and complete the tasks. This statement is related to the gamification features in WReSTTCyLE.

\subsection{Discussion}

Based on the results presented in the previous section, the instructors generally thought that attending the workshops had a positive impact on the classes taught post-workshops. More specifically, the workshops provided the instructors with the knowledge of how to integrate testing into their courses. A majority of the instructors who responded to the survey used WReSTT-CyLE in the classroom. In addition, a majority of instructors thought that WReSTT-CyLE would be useful teaching resource for their colleagues. The results obtained from the students who responded to the survey were also positive. Student overall experience with using WReSTT-CyLE was reported as being positive. The testing tutorials and DLOs in WReSTT-CyLE was rated as good, however the students would have liked to see more tutorials available in WReSTT-CyLE. The use of the LESs appeared to have a positive impact on how student interacted with WReSTT-CyLE.

The main area of concern with administering the surveys was the response rates. The response rate were particularly low for the students who used the system during the three year period under consideration. There are several factors that affected the low response rate for the student survey. The main factor being students leaving the university and not using the email we have for them on file. It should be noted that only 75 email survey requests out of 1055 bounced back, this would lead us to conclude that a majority of the requests were either ignored or were not read. We plan to provide some incentives for students to complete the surveys going forward. We also plan to administer the survey well before the end of the semester.

One other limitation of the study is that it is very possible that those who responded to the surveys are different from those who did not respond. For example, for the instructor survey, it may be that only those instructors who viewed the workshops as helpful actually responded. Those who did not respond may have been more likely to find that the workshops were not helpful and did not incorporate any of the knowledge from the workshop into their courses. It should also be noted that the study involved self-reported surveys and has the reliability and limitations of such surveys, e.g., potential positive response bias for people who do respond to the questionnaire. Based on our observations from the workshops, the instructors thought that the learning content was too advance for the introductory programming courses. A majority of the instructors who attended the workshops taught CS1/2 courses. To remedy this situation we are currently extending the content to include CS1/2 concepts and testing content targeted for lower level programming courses.

\section{Related Work}

There are several projects and repositories that support the teaching of software testing and provide instructors with learning materials. There is also work on how LESs can be integrated into teaching courses in the CS/IT discipline. 
Li et al. ${ }^{12}$ found that gamification can be used to increase engagement of CS students in an online, social network-based, collaborative learning environment (PeerSpace). The game mechanics used in PeerSpace include: participant points, participation levels, and leader boards. Liccardi et al. ${ }^{13}$ investigated the role social networks in computer science education. It was found that social interaction within an online framework can help university students share experiences and collaborate on relevant topics. As such, social networks can act as a pedagogical agent, for example, with problem-based learning. Cassel et al. ${ }^{2}$ coordinate the activities of Ensemble - Computing Portal which is a NSF sponsored National Science Digital Library (NSDL) Pathways project for computing education materials, and provides access to other repositories, e.g., YouTube Education.

Several educators and researchers have done work on integrating testing into CS/IT courses throughout the college curriculum. Wong ${ }^{20}$ describes an approach that integrates software testing throughout the CS curriculum starting from CS1 through to the final senior project course. The approach emphasizes introducing testing modules (possibly the same module) into several courses in the sequence (varying the breath and depth of delivery) using a minimally intrusive and non-restrictive way. The approach provides the instructor with the ability to introduce the testing modules that is more accessible and understandable to the students. Edwards et al. ${ }^{6}$ develop Web-CAT: the Webbased Center for Automated Testing provides a set of services that support test-driven development. Web-CAT allows students to submit their code and test cases and obtain various results.

The authors of this paper have publish several works with studies using WReSTT-CyLE and it forerunners. ${ }^{3,4,7}$ These studies focused on studies conducted at a single institution and in some cases not using all the LESs. In this paper we report on how WReSTT-CyLE is used at multiple intuitions and report on the first post-workshop survey administered in the project. WReSTT-CyLE is different to other repositories like PeerSpace and Ensemble since the learning content is presented using a combination of LESs - collaborative learning, gamification and social interaction. Although the LESs described in this paper are used in varying ways in existing Learning Management Systems (LMSs), e.g., Moodle, ${ }^{16}$ the use of LESs in WReSTT-CyLE is different. That is, WReSTT-CyLE integrates the LESs in such a way to motivate students to visit the site and encourage student teams to participate in the team activities. ${ }^{4}$ In addition, the overhead for instructors to access vetted learning content (DLOs and tool tutorials) is reduced since they already exist in WReSTT-CyLE.

\section{Conclusions}

In this paper we briefly describe the new features in WReSTT-CyLE Web-Based Repository of Software Testing Tutorials: a Cyberlearning Environment, report on a results of a 4-year project that involved instructor professional development and using WReSTT-CyLE in the classroom. WReSTT-CyLE uses a combination of learning and engagement strategies (LESs) that motivates students to visit and access the learning material (learning object and tutorials) in WReSTT-CyLE. These LESs include collaborative learning, gamification, and social interaction. The results of the project showed that there was a positive impact on the classes taught by those instructors that attended the professional development workshops. In addition, the results of this paper and other publications related to the project showed that students overall experience with using WReSTTCyLE was positive. The main suggested improvement of WReSTT-CyLE is to increase the number of learning objects and tutorials targets for CS1/2 classes. 


\section{Acknowledgments}

We would like to thank the reviewers for their valuable comments. This work was supported in part by the National Science Foundation under grants DUE-0736833 and DUE-1225742 (FIU), DUE-1225654 (AAMU), DUE-1224411 (MU) and DUE-1225972 (NDSU).

\section{References}

${ }^{1}$ P. Bourque and R. Dupuis. Guide to the Software Engineering Body of Knowledge 2004 Version. IEEE Computer Society, Los Alamitos, California, 2004.

${ }^{2}$ L. Cassel, L. Delcambre, E. Fox, and R. Furuta. Ensemble - Computing Portal Connecting Computing Educators, February 2017. http: //www . computingportal .org/.

${ }^{3}$ P. J. Clarke, D. Davis, R. Chang-lau, and T. King. Observations on student use of tools in an undergraduate testing class. In 121st ASEE Annual Conference and Exposition. ASEE, 2014. Paper No. 10123.

${ }^{4}$ P. J. Clarke, D. Davis, T. M. King, J. Pava, and E. L. Jones. Integrating testing into software engineering courses supported by a collaborative learning environment. Transactions on Computing Education, 14(3):18:1-18:33, Oct. 2014.

${ }^{5}$ Drupal Community. Drupal version 8, November 2015. https: / /www. drupal . org/8 (retrievedFeb. 2017 ).

${ }^{6}$ S. Edwards. Web-CAT: the Web-based Center for Automated Testing, February 2017. http://web-cat . org/.

${ }^{7}$ Y. Fu and P. J. Clarke. Gamification based cyber enabled learning environment of software testing. In ASEE Annual Conference \& Exposition. ASEE, 2016. Paper No. 15359.

${ }^{8}$ E. Gamma and K. Beck. JUnit, January 2017. http: / / www . junit. org / (retrieved Jan. 2017).

${ }^{9}$ M. R. Hoffmann, B. Janiczak, and E. Mandrikov. EclEmma, February 2015. http: / / www . eclemma . org/.

${ }^{10}$ IBM. Rational Functional Tester. http://www-03.ibm.com/software/products/en/ functional (retrievedJan.2017).

${ }^{11}$ IEEE Learning Technology Standards Committee (LTSC). Ieee standard for learning object metadata. IEEE Std 1484.12.1-2002, pages i-32, 2002.

${ }^{12}$ C. Li, Z. Dong, R. H. Untch, and M. Chasteen. Engaging computer science students through gamification in an online social network based collaborative learning environment. International Journal of Information and Education Technology, 3(1):72 - 77, 2013.

${ }^{13}$ I. Liccardi, A. Ounnas, R. Pau, E. Massey, P. Kinnunen, S. Lewthwaite, M.-A. Midy, and C. Sarkar. The role of social networks in students' learning experiences. SIGCSE Bull., 39(4):224-237, Dec. 2007.

${ }^{14}$ T. W. Malone. What makes things fun to learn? heuristics for designing instructional computer games. In Proceedings of the 3rd ACM SIGSMALL Symposium and the First SIGPC Symposium on Small Systems, SIGSMALL '80, pages 162-169, New York, NY, USA, 1980. ACM.

15 A. P. Mathur. Foundations of Software Testing. Addison-Wesley Professional, 1st edition, 2008.

${ }^{16}$ Moodle Development Team. Moodle, Dec. 2016. https: //moodle. org/.

${ }^{17}$ Qualtrics Team. Qualtrics, Feb. 2017.

${ }^{18}$ B. L. Smith and J. T. MacGregor. What is Collaborative Learning? In A. Goodsell, M. Maher, and V. Tinto, editors, Collaborative Learning: A Sourcebook for Higher Education. National Center on Postsecondary Teaching, Learning, and Assessment, University Park, Pa., 1992.

${ }^{19}$ R. S. Smith. Guidelines for authors of learning objects. The New Media Consortium, 2004.

${ }^{20}$ W. E. Wong. Improving the state of undergraduate software testing education. In 2012 American Society for Engineering Education (ASEE) Annual Conference \& Exposition. ASEE, June 2012. https://peer. asee. org/21511. 